\title{
First Results from CMS on QCD, Quarkonia, and Heavy-Flavour Physics in proton-proton Collisions at $\sqrt{s}=7 \mathrm{TeV}$
}

\section{Sara Bolognesi (CERN), on behalf of the CMS Collaboration}

E-mail: sara.bolognesi@cern.ch

\begin{abstract}
The very good performance of the CMS detector [1] allows to establish a rich set of physics measurements with the first few hundred $\mathrm{nb}^{-1}$ of LHC pp-collision data collected at $\sqrt{s}=7 \mathrm{TeV}$. The first particle multiplicity measurements can be used to tune the Underlying Event description in Parton Shower Monte-Carlo (MC). Given the advanced level of jet commissioning, the differential cross-section of inclusive jet production has been measured from $20 \mathrm{GeV}$ to $500 \mathrm{GeV}$ in transverse momentum of the leading jet. Measurements of the hadronic event shapes have the sensitivity to tune the Matrix Element MC. B-tagging techniques can be exploited to measure the inclusive b-jet cross-section. The b-jet $\rightarrow \mu+X$ cross-section is also measured, showing an underestimation of the Next-to-Leading-Order (NLO) calculations at very low $p_{T}$ and central pseudorapidity $(\eta)$. Finally, first measurements of the $J / \psi \rightarrow \mu \mu$ and $\Upsilon \rightarrow \mu \mu$ cross-sections were performed, thanks to the high muon trigger efficiency and very good tracking resolution, and compared with theoretical models for quarkonia production.
\end{abstract}

35th International Conference of High Energy Physics

July 22-28, 2010

Paris, France

${ }^{*}$ Speaker. 


\section{QCD}

The measurement of particle multiplicity [2] is performed with three independent methods: pixel clusters, tracklets and tracks counting, the last one being able to go down to $50 \mathrm{MeV}$ in $p$. The measurement is corrected to Non-Single-Diffractive component and the systematics related to this correction (2.5\%) is the dominant one. The results at $0.9 \mathrm{TeV}$ are well in agreement with previous experiments while the increasing of multiplicity at $2.36 \mathrm{TeV}$ and $7 \mathrm{TeV}$ is underestimated by the available MC tunes. This result is also confirmed by the analysis of the underlying event in the transverse region [3]. At low value of $p_{T}$ of the leading track (or jet), the particle multiplicity is underestimated by present MC tunes and the increase of the hadronic activity from 0.9 to $7 \mathrm{TeV}$ is not properly described.

Four different jet reconstruction algorithms are available in CMS [4]: calorimetric only (CaloJets), calorimetric complemented with tracking information (Jets-Plus-Tracks), tracks only (TrackJets) and Particle Flow (PF-Jets). Results from different jet types are in good agreement within the systematic and statistical uncertainty. The jet energy corrections are currently taken from MC, because of lack of statistics in data, but all the ingredients are cross-checked in details with data [4]. The uncertainty on the absolute (relative) jet energy scale is $10 \%$ on calorimetric jets and $5 \%$ for the other types of jets (2\% linearly increasing with $\eta$ ). The anti- $k_{T}$ algorithm [5] is used. Mainly a single jet trigger with $15 \mathrm{GeV}$ threshold is exploited.

The measured di-jet mass spectrum [6] contains events up to about $2 \mathrm{TeV}$ and it is in agreement with QCD expectations. The di-jet angular distribution is probed [7] by the $\chi_{\text {dijet }}=\exp \left(\left|y_{1}-y_{2}\right|\right)$ variable, where $y_{1,2}$ are the rapidities of the two leading jets: the agreement with NLO computation is good. The di-jet azimuthal decorrelation between the two leading jets $\Delta \phi_{\text {lijet }}=\left|\phi_{\text {jet } 1}-\phi_{\text {jet } 2}\right|$, which is sensitive to higher order QCD radiation effects, is measured and compared with MC predictions [7]: MadGraph [8] tends to underestimate the multi-jet (low $\Delta \phi$ ) region.

The hadronic event shapes (central transverse thrust and central trust minor) are measured [10] to test the transition region between two- and three-jets production. The main systematic uncertainty is due to the jet energy scale but the sensitivity is sufficient for a first tuning of Alpgen [9] and Madgraph [8]. The ratio of events with two and three jets is also measured directly [11]: the results are in agreement both with MadGraph [8] and Pythia [12] within the actual uncertainties.

Finally the differential jet cross-section is measured in various rapidity bins (up to $|y|<3$ ) [13]. Events from single jet triggers with different thresholds are merged together with minimum bias events. With Particle Flow techniques, jets down to $18 \mathrm{GeV}$ can be identified, as shown in Fig. 1. The spectrum is corrected with a resolution unfolding procedure and the main systematic is due to luminosity (11\%, common to all the cross-section measurements in CMS with the present statistics) and to the jet energy scale $(\sim 10 \%)$. The results are well in agreement with the NLO predictions, given the experimental and theoretical uncertainties.

\section{Inclusive B-physics}

The inclusive $\mathrm{b}$ production cross-section is measured exploiting a b-tagging technique based on secondary vertex identification [14]. The b-tagging purity and efficiency are currently taken from MC and the final systematic on the latter is $20 \%$, due to the small currently available statis- 

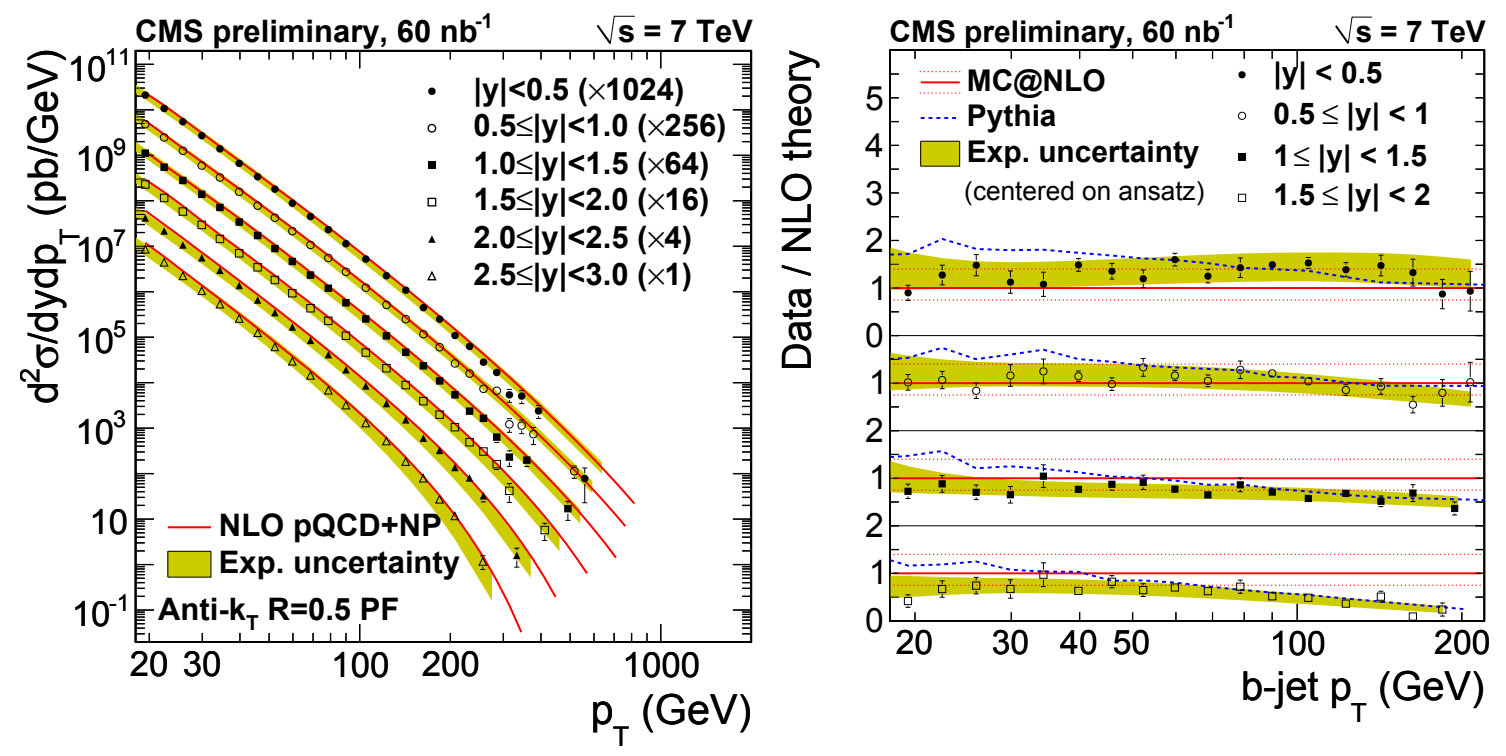

Figure 1: Left: unfolded measured spectra of PF-jets compared with theory predictions (different $|y|$ bins are multiplied by arbitrary factors for better visibility). Right: ratio of unfolded measured spectra of PF b-jets over MC@NLO predictions.

tics for data-driven cross-checks. The measurement is done similarly to the inclusive jet analysis described in Sec. 1. The differential cross-section in different rapidity bins (up to $|y|<2$ ) is in reasonable agreement with MC@NLO [15] but, as shown in Fig. 1, there are shape differences in $p_{T}$ and $\eta$. Similar results are obtained for the ratio between b- and inclusive jet cross-sections.

The b-jet $\rightarrow \mu+X$ cross-section as a function of the muon $p_{T}$ (down to $6 \mathrm{GeV}$ ) is measured [16] through a fit to the distribution of the muon $p_{T}$ relative to the jet axis $\left(p_{T}^{\text {rel }}\right)$. The lightflavors template is taken from data while the $\mathrm{b}$ and $\mathrm{c}$ templates from MC but validated with a datadriven method. The main systematics are due to the uncertainties on the templates shape, on the jet reconstruction efficiency and on the luminosity. The results are compared with MC@NLO [15] predictions: the cross-section is underestimated by the theory in the low $p_{\Gamma}$ and central $|\eta|$ region.

\section{Quarkonia}

The high muon trigger efficiency [17] and the very good tracking scale and resolution [18] are the basis of the $J / \psi \rightarrow \mu \mu$ and $\Upsilon \rightarrow \mu \mu$ cross-section measurements [19, 20]. The analysis is performed separately in the barrel region, where the resolution is better and the background lower, and in the endcap region where the acceptance reaches down to zero $p_{T}$. The largest systematic is due to the statistical uncertainty on the efficiency measured from data with the tag-and-probe technique. Since the polarization of $J / \psi$ and $\Upsilon$ is basically unknown and the acceptance has a strong dependence on it, the results must be quoted separately for five different polarization scenarios.

In the $J / \psi$ case the prompt and non-prompt components are separated through a fit to the decay length distribution of displaced $J / \psi$ decays. The $B \rightarrow J / \psi$ cross-section is in good agreement with Fixed-Order-Next-to-Leading-Log computation [21] while the prompt cross-section is not in agreement at low $p_{T}$ with any of the available theoretical models. 
The $\Upsilon(1 S)$ cross-section is in reasonable agreement with the Color Evaporation Model [22]. Finally the ratio $(\Upsilon(2 S)+\Upsilon(3 S)) / \Upsilon(1 S)$ has been measured to be $0.44 \pm 0.06$ (stat) \pm 0.07 (syst)

\section{References}

[1] CMS Collaboration, The CMS experiment at the CERN LHC, JINST 3 S08003 (2008)

[2] CMS Collaboration, Charged particle multiplicities in pp interactions at $\sqrt{s}=0.9,2.36$, and 7.0 TeV, CMS PAS QCD-10-004

[3] CMS Collaboration, Measurement of the Underlying Event Activity at the LHC with $\sqrt{s}=7 \mathrm{TeV}$ and Comparison with $\sqrt{s}=0.9 \mathrm{TeV}$, CMS PAS QCD-10-010

[4] CMS Collaboration, Jet Performance in pp Collisions at 7 TeV, CMS PAS JME-10-003

[5] M. Cacciari, G. P. Salam, and G. Soyez, The anti-kt jet clustering algorithm, JHEP 0804:063 (2008)

[6] CMS Collaboration, Search for Dijet Resonances in the Dijet Mass Distribution in pp Collisions at $\sqrt{s}=7 \mathrm{Te}$, CMS PAS EXO-10-001

[7] CMS Collaboration, Dijet Azimuthal Decorrelations and Angular Distributions in pp Collisions at $\sqrt{s}=7 \mathrm{Te} V$, CMS PAS QCD-10-015

[8] J. Alwall et al., MadGraph/MadEvent v4: The NewWeb Generation, JHEP 09 (2007) 028

[9] M. L. Mangano, M. Moretti, F. Piccinini et al., ALPGEN, a generator for hard multiparton processes in hadronic collisions, JHEP 07 (2003) 001, arXiv:hep-ph/0206293.

[10] CMS Collaboration, Hadronic Event Shapes in pp Collisions at 7 TeV, CMS PAS QCD-10-013

[11] CMS Collaboration, Measurement of the 3-jet to 2-jet Cross Section Ratio in pp Collisions at $\sqrt{s}=7 \mathrm{Te}$, CMS PAS QCD-10-012

[12] T. Sjostrand, S. Mrenna, and P. Skands, PYTHIA 6.4 physics and manual, JHEP 0605 (2006) 026

[13] CMS Collaboration, Measurement of the Inclusive Jet Cross Section in pp Collisions at 7 TeV, CMS PAS QCD-10-011

[14] CMS Collaboration, Inclusive b-jet production in pp collisions at $\sqrt{s}=7 \mathrm{TeV}$, CMS PAS BPH-10-009

[15] S. Frixione, P. Nason, and B. R.Webber, Matching NLO QCD and parton showers in heavy flavour production, JHEP 08 (2003) 007, arXiv:hep-ph/0305252.

[16] CMS Collaboration, Open beauty production cross section with muons in pp collisions at a center-of-mass energy of $7 \mathrm{TeV}$, CMS PAS BPH-10-007

[17] CMS Collaboration, Performance of muon identification in pp collisions at $\sqrt{s}=7 \mathrm{TeV}$, CMS PAS MUO-10-002

[18] CMS Collaboration, Measurement of Momentum Scale and Resolution of the CMS Detector using Low-mass Resonances and Cosmic Ray Muons, CMS PAS TRK-10-004

[19] CMS Collaboration, J/Psi prompt and non-prompt cross sections in pp collisions at $\sqrt{s}=7 \mathrm{TeV}$, CMS PAS BPH-10-002

[20] CMS Collaboration, Measurement of the Inclusive Upsilon production cross section in pp collisions at $\sqrt{s}=7 \mathrm{TeV}$, CMS PAS BPH-10-003

[21] M. Cacciari et al., QCD analysis of first b cross section data at $1.96 \mathrm{TeV}$, JHEP 0407 (2004) 033.

[22] V. D. Barger,W.-Y. Keung, and R. J. N. Phillips, On psi and Upsilon Production via Gluons, Phys. Lett. B91 (1980) 253. [doi:10.1016/0370-2693(80)90444-X]. 